\title{
The algebra generated by a pair of operator weighted shifts
}

\author{
by MAREK PTAK (Kraków)
}

\begin{abstract}
We present a model for two doubly commuting operator weighted shifts. We also investigate general pairs of operator weighted shifts. The above model generalizes the model for two doubly commuting shifts. WOT-closed algebras for such pairs are described. We also deal with reflexivity for such pairs assuming invertibility of operator weights and a condition on the joint point spectrum.
\end{abstract}

1. Introduction. In what follows $L(\mathbf{H})$ denotes the algebra of all (linear, bounded) operators in a complex separable Hilbert space $\mathbf{H}$ and $I_{\mathbf{H}}$ or $I$ stands for the identity in $\mathbf{H}$. By an algebra of operators we always mean a $\operatorname{WOT}(=$ weak operator topology)-closed subalgebra of $L(\mathbf{H})$ with unit $I_{\mathbf{H}}$. If $\mathcal{S} \subset L(\mathbf{H})$, then $\mathcal{W}(\mathcal{S})$ and Lat $\mathcal{S}$ stand for the WOT-closed algebra generated by $\mathcal{S}$ and the lattice of all (closed) invariant subspaces for $\mathcal{S}$, respectively. $\operatorname{Alg}$ Lat $\mathcal{S}$ stands for the algebra of all operators on $\mathbf{H}$ which leave invariant all subspaces from Lat $\mathcal{S}$. An algebra $\mathcal{W}$ is called reflexive if $\mathcal{W}=\operatorname{Alg}$ Lat $\mathcal{W}$. A family $\mathcal{S} \subset L(\mathbf{H})$ is called reflexive if so is $\mathcal{W}(\mathcal{S})$. Operators $T_{1}, T_{2} \in \mathbf{H}$ doubly commmute if $T_{1}$ commutes with $T_{2}$ and $T_{1}$ commutes with $T_{2}^{*}$.

In the paper we present a model for two doubly commuting operator weighted shifts (Section 2) which generalizes a model for two doubly commuting (but not operator or weighted) shifts (see Theorem 1 of [6]). In [4], the general pair of (neither operator nor weighted) shifts was considered. The main purpose of the paper is to investigate general pairs of operator weighted shifts (for definition see Section 3). This generalizes two doubly commuting operator weighted shifts, in view of the model given in Section 2. On the other hand, a special case of a general pair of scalar weighted shifts was considered in [1]. In what follows we describe the WOT-closed algebra generated by a pair of operator weighted shifts (Theorem 6.2). We also deal

1991 Mathematics Subject Classification: Primary 47D25.

Key words and phrases: pairs of operator weighted shifts, reflexive algebras. 
with reflexivity for such a pair (Theorem 6.7) assuming invertibility of operator weights and a condition on the joint point spectrum. The corresponding results for a single operator weighted shift were given by Lambert [2].

In Section 4 we present basic properties of the joint point spectrum and joint eigenvalues. In Section 5 we present the case of pairs of operator weighted shifts "defined on the pairs of non-negative integers". In view of the model given in Section 2, it describes two doubly commuting operator weighted shifts. Section 6 discusses the general case. Examples are given in Section 7.

In what follows, let $G_{0}$ denote the set of all pairs of non-negative integers and $G$ be the set of all pairs of integers. If $\phi=\left(\phi^{(1)}, \phi^{(2)}\right), \psi=\left(\psi^{(1)}, \psi^{(2)}\right) \in$ $G$, then we write $\phi \leq \psi$ if and only if $\phi^{(1)} \leq \psi^{(1)}, \phi^{(2)} \leq \psi^{(2)}$. Let $\varepsilon_{1}=(1,0)$, $\varepsilon_{2}=(0,1)$.

\section{Model for two doubly commuting operator weighted shifts.} The main result of this section is

Proposition 2.1 Let $T_{1}, T_{2} \in L(\mathbf{K})$ be operator weighted shifts whose weights have dense ranges. Assume that $T_{1}, T_{2}$ doubly commute. Then there is a wandering subspace $L$ and families $\left\{A_{\phi}^{(l)}: \phi \in G_{0}\right\}(l=1,2)$ such that $\mathbf{K}=\bigoplus_{\phi \in G_{0}} L_{\phi}$, where $L_{\phi}=L$ and for $f=\sum_{\phi \in G_{0}} f_{\phi} e_{\phi}, f_{\phi} \in L$ and $e_{\phi}$ indicates that $f_{\phi} e_{\phi}$ is an element of $L_{\phi}$, we have

$$
T_{i} f=\sum_{\phi \in G_{0}}\left(A_{\phi}^{(i)} f_{\phi}\right) e_{\phi+\varepsilon_{i}} \quad \text { for } i=1,2 .
$$

Moreover,

$$
\overline{T_{1}^{i} T_{2}^{j} L_{(0,0)}}=L_{(i, j)} \quad \text { for all }(i, j) \in G_{0}
$$

and

$$
\begin{aligned}
A_{\phi+\varepsilon_{2}}^{(1)} A_{\phi}^{(2)} & =A_{\phi+\varepsilon_{1}}^{(2)} A_{\phi}^{(1)}, \\
A_{\phi}^{(1)} A_{\phi}^{(2) *} & =A_{\phi+\varepsilon_{1}}^{(2) *} A_{\phi+\varepsilon_{2}}^{(1)}, \\
A_{\phi}^{(2)} A_{\phi}^{(1) *} & =A_{\phi+\varepsilon_{2}}^{(2) *} A_{\phi+\varepsilon_{1}}^{(1)} \quad \text { for all } \phi \in G_{0} .
\end{aligned}
$$

Proof. Since $T_{1}$ is a weighted shift, there is a subspace $H$ such that $\mathbf{K}=\bigoplus_{i=0}^{\infty} H_{i}$, where $H_{i}=H$, and for $f=\sum_{i=0}^{\infty} f_{i} e_{i} \in \mathbf{K}, T_{1} f=$ $\sum_{i=0}^{\infty} B_{i}^{(1)} f_{i} e_{i+1}$ for some bounded family of operators $\left\{B_{i}^{(1)} \in L(H): i=\right.$ $1,2, \ldots\}$. The operator $T_{2}$ can be represented as a matrix, say $\left[X_{i j}\right]$. Thus by Lemma 2.1 of $[3], X_{i j}=0$ for $i<j$. The operator $T_{2}^{*}$ is represented by $\left[X_{j i}^{*}\right]$. It also commutes with $T_{1}$, and thus $X_{i j}^{*}=0$ for $i>j$. Hence $X_{i j}=0$ for $i \neq j$. Therefore $H_{i}$ reduces $T_{2}$ for all $i$.

The operators $\left.T_{2}\right|_{H_{i}}=X_{i i}$, for all $i$, are weighted shifts, and thus there are subspaces $L^{(i)}$ such that $H_{i}=\bigoplus_{j=0}^{\infty} L_{(i, j)}$, where $L_{(i, j)}=L^{(i)}$, and 
for $f^{(i)}=\sum_{j=0}^{\infty} f_{j}^{(i)} e_{j}^{(i)} \in H_{i},\left.T_{2}\right|_{H_{i}} f^{(i)}=\sum_{j=0}^{\infty} B_{(i, j)}^{(2)} f_{j}^{(i)} e_{j+1}^{(i)}$ for some bounded families of operators $\left\{B_{(i, j)}^{(2)} \in L\left(L^{(i)}\right): j=1,2, \ldots\right\}$.

Now we prove that $T_{1} L_{(i, 0)} \subset L_{(i+1,0)}$. Assume that, on the contrary, there is $x \in L_{(i, 0)}$ such that $\left(T_{1} x,\left.T_{2}^{j}\right|_{H_{i+1}} y\right) \neq 0$ for some $y \in H_{i+1}, j>0$. Then

$$
\left(T_{1} x,\left.T_{2}^{j}\right|_{H_{i+1}} y\right)=\left(T_{2}^{j *} T_{1} x, y\right)=\left(T_{1} T_{2}^{j *} x, y\right)=0
$$

since $x \in L_{(i, 0)}$ and $T_{2}^{*} x=0$. Hence $T_{1} L_{(i, 0)} \subset L_{(i+1,0)}$.

Thus $T_{1} T_{2}^{j} L_{(i, 0)}=T_{2}^{j} T_{1} L_{(i, 0)} \subset T_{2}^{j} L_{(i+1,0)} \subset L_{(i+1, j)}$. The weights of $T_{2}$ have dense ranges, so $\overline{T_{2}^{j} L_{(i, 0)}}=L_{(i, j)}$. Hence $T_{1} L_{(i, j)} \subset L_{(i+1, j)}$.

To prove (2.2), assume first that there is $0 \neq y \in L_{(i+1,0)}$ such that $T_{1} L_{(i, 0)} \perp y$. Then $T_{2}^{*} y=0$ and $T_{1}^{*} y \neq 0$ since $y \in H_{i+1}$ and $\operatorname{ker} B_{i}^{(1) *}=\{0\}$. Also, $T_{1}^{*} y \in H_{i}$ and $T_{1}^{*} y \perp L_{(i, 0)}$. This implies that $T_{2}^{*} T_{1}^{*} y \neq 0$ since $\operatorname{ker} B_{(i, j)}^{(2) *}=\{0\}$ for $j=0,1, \ldots$ Then $T_{1}^{*} T_{2}^{*} y \neq 0$, contrary to $T_{2}^{*} y=0$. Thus $\overline{T_{1} L_{(i, 0)}}=L_{(i+1,0)}$. Hence $\overline{T_{1}^{i} L_{(0,0)}}=L_{(i, 0)}$. We also have $\overline{T_{2} L_{(i, j)}}=L_{(i, j+1)}$, since weights of $T_{2}$ have dense ranges. Hence $\overline{T_{1}^{i} T_{2}^{j} L_{(0,0)}}=L_{(i, j)}$.

Since $H_{i_{1}}=H_{i_{2}}$, we have $L^{\left(i_{1}\right)}=L^{\left(i_{2}\right)}=: L$. Define $A_{(i, j)}^{(1)}=\left.B_{i}^{(1)}\right|_{L_{(i, j)}}$ and $A_{(i, j)}^{(2)}=B_{(i, j)}^{(2)}$. It is easy to see that (2.1) is satisfied. The operators $T_{1}, T_{2}$ doubly commute, so (2.1) implies (2.3).

In Corollary 5.6 we will consider reflexivity of the above.

3. Definition and elementary properties of a pair of operator weighted shifts. Following [4], we introduce some notation and definitions. Namely, a subset $X \subset G$ is called a diagram if $\phi \in X, s \in G_{0}$ implies $\phi+s \in X$. The set of all diagrams is denoted by $\mathbf{X}$. For $\phi \in G$ we define $E_{\phi}=\{X \in \mathbf{X}: \phi \in X\}$. It is obvious that $E_{\phi} \subset E_{\phi+s}\left(\phi \in G, s \in G_{0}\right)$. Let $\mathcal{B}$ be the $\sigma$-algebra generated by $E_{\phi}(\phi \in G)$ and $\mu$ be a positive finite measure on $(\mathbf{X}, \mathcal{B})$.

Set $\mathcal{H}=\bigoplus_{\phi \in G} H_{\phi}$, where $H_{\phi}=\mathbf{H}$ and consider the space $\mathbf{K}$ of all measurable functions $f: \mathbf{X} \rightarrow \mathcal{H}$ such that $\int\|f(X)\|^{2} d \mu \leq \infty$ and $f(X) \in$ $\bigoplus_{\phi \in X} H_{\phi}$ (we identify functions equal $\mu$-a.e.). Then $\mathbf{K}$ is a Hilbert space with inner product $(f, g)=\int(f(X), g(X))_{\mathcal{H}} d \mu(X)$. Each element of $\mathbf{K}$ can be written as $f=\sum_{\phi \in G} f_{\phi}(\cdot) e_{\phi}$, where $f_{\phi} \in \mathcal{H}_{\phi}:=\left\{f \in L^{2}(\mathbf{X}, \mathcal{B}, \mu, \mathbf{H})\right.$ : $f_{\phi}(X)=0 \mu$-a.e. on $\left.X-E_{\phi}\right\}$, and $e_{\phi}$ indicates that $f_{\phi}(X) e_{\phi}$ is an element of $H_{\phi}$ for $X \in \mathbf{X}$.

Let $\left\{A_{\phi}^{(i)}(\cdot): \mathbf{X} \rightarrow L(\mathbf{H}), A_{\phi}^{(i)}(X)=0 \mu\right.$-a.e. on $\left.X-E_{\phi}, \phi \in G\right\}(i=$ $1,2)$ be sets of uniformly bounded operator functions $\left(\sup \left\{\operatorname{supess}\left\|A_{\phi}^{(i)}(\cdot)\right\|\right.\right.$ : $\phi \in G, i=1,2\}=C<\infty)$. We will consider $A_{\phi}^{(i)}(\cdot)$ as operators on $\mathcal{H}_{\phi}$. In 
what follows we assume that $\operatorname{ker} A_{\phi}^{(i)}(\cdot)=\{0\}, \phi \in G, i=1,2$. Hence we can define operators $T_{1}, T_{2}$ on $\mathbf{K}$. If $f=\sum_{\phi \in G} f_{\phi}(\cdot) e_{\phi}$ then

$$
T_{i} f=\sum_{\phi \in G} A_{\phi}^{(i)}(\cdot) f_{\phi}(\cdot) e_{\phi+\varepsilon_{i}}, \quad i=1,2 .
$$

Since $\mathbf{X}$ is the set of diagrams, we have $T_{i} f \in \mathbf{K}, i=1,2$. The sets $\left\{A_{\phi}^{(i)}(\cdot)\right\}, i=1,2$, are uniformly bounded, and thus $T_{1}, T_{2} \in L(\mathbf{K})$. Let us call $\left\{T_{1}, T_{2}\right\}$ a pair of operator weighted shifts.

Lemma 3.1. The operators $T_{1}, T_{2}$ commute if and only if

$$
A_{\phi+\varepsilon_{2}}^{(1)}(\cdot) A_{\phi}^{(2)}=A_{\phi+\varepsilon_{1}}^{(2)}(\cdot) A_{\phi}^{(1)}(\cdot) \quad \text { for all } \phi \in G .
$$

To prove the lemma, it is enough to compare $T_{1} T_{2}$ with $T_{2} T_{1}$ on an element of $\mathbf{K}$. From now on we assume that $T_{1}, T_{2}$ commute.

For $s=\left(s_{1}, s_{2}\right) \in G_{0}$ and $\phi \in G$ let $T^{s}=T_{1}^{s_{1}} T_{2}^{s_{2}}$ and

$$
\begin{aligned}
S_{\phi}^{s}(\cdot)= & A_{\phi+s-\varepsilon_{2}}^{(2)}(\cdot) \ldots A_{\phi+s_{1} \varepsilon_{1}+\varepsilon_{2}}^{(2)}(\cdot) A_{\phi+s_{1} \varepsilon_{1}}^{(2)}(\cdot) \\
& \cdot A_{\phi+\left(s_{1}-1\right) \varepsilon_{1}}^{(1)}(\cdot) \ldots A_{\phi+\varepsilon_{1}}^{(1)}(\cdot) A_{\phi}^{(1)}(\cdot) .
\end{aligned}
$$

Hence, for $f=\sum_{\phi \in G} f_{\phi}(\cdot) e_{\phi}$,

$$
T^{s} f=\sum_{\phi \in G} S_{\phi}^{s}(\cdot) f_{\phi}(\cdot) e_{\phi+s} .
$$

Now, if $\phi \in G$ then we write $G_{\phi}=\phi+G_{0}=\left\{\phi+s: s \in G_{0}\right\}$ and $L_{\phi}=\left\{f \in \mathbf{K}: f(X) \in \bigoplus_{\psi \in G_{\phi}} H_{\psi} \mu\right.$-a.e. and $f(X)=0 \mu$-a.e. on $\left.X-E_{\phi}\right\}$.

Let us recall from [4] that

Remark 3.2. $\mathbf{K}=\overline{\operatorname{span}\left\{L_{\phi}: \phi \in G\right\}}$.

The following lemma can be proved similarly to Lemma 5 of [4].

Lemma 3.3. The subspace $L_{\phi}$ is invariant for $T^{s}$ if $s \in G_{0}$ and $\phi \in G$.

Let us state the basic examples.

Example 3.4. Let $X$ be a diagram and $\mu=\delta_{X}$ (the point mass at $X$ ). Then the sets $\left\{A_{\phi}^{(i)}\right\}_{\phi \in X} \subset L(\mathbf{H}), i=1,2$, generate operators $T_{1}, T_{2}$ on the spaces $\mathbf{K}_{X}:=\left\{f: f=\sum_{\phi \in X} f_{\phi} e_{\phi}, f_{\phi} \in \mathbf{H}\right\}$.

EXAmple 3.5. In view of Proposition 2.1, notice the special case of Example 3.4 with $X=G_{0}$, since it is a model for two doubly commuting weigted shifts. In that case, we write $\mathbf{K}_{0}$ instead of $\mathbf{K}_{G_{0}}$.

4. Joint eigenvalues. We start with recalling the definition of the joint eigenvalue. Let $B_{1}, B_{2} \in L(\mathbf{H})$. Then we write $\lambda=\left(\lambda_{1}, \lambda_{2}\right) \in \sigma_{\mathrm{p}}\left(B_{1}, B_{2}\right)$ if there exists a non-zero vector $x \in \mathbf{H}$ such that $\left(B_{i}-\lambda_{i}\right) x=0$ for $i=1,2$. It is easy to see that $\mu\left(E_{\phi}\right) \neq 0$ implies $0 \in \sigma_{\mathrm{p}}\left(\left(\left.T_{1}\right|_{L_{\phi}} ^{*},\left(\left.T_{2}\right|_{L_{\phi}}\right)^{*}\right)\right.$ for all $\phi \in G$. 
Now we turn our attention to Example 3.5 and consider the operators $T_{1}, T_{2} \in L\left(\mathbf{K}_{0}\right)$ given there. As for a single shift, we can show

Lemma 4.1. Let $\lambda=\left(\lambda_{1}, \lambda_{2}\right)$ be a non-zero joint eigenvalue for $T_{1}^{*}, T_{2}^{*}$. Then $\lambda^{\prime}=\left(\lambda_{1}^{\prime}, \lambda_{2}^{\prime}\right)$ is a joint eigenvalue for $T_{1}^{*}, T_{2}^{*}$ if $\left|\lambda_{i}^{\prime}\right| \leq\left|\lambda_{i}\right|$ for $i=1,2$.

It is easy to show the following

Lemma 4.2. Let $T_{1}, T_{2}$ be as in Example 3.5 and let $f=\sum_{\psi \in G_{0}} f_{\psi} e_{\psi} \in$ $\mathbf{K}_{0}$. Then $\lambda=\left(\lambda_{1}, \lambda_{2}\right)$ is a joint eigenvalue for $T_{1}^{*}, T_{2}^{*}$ with a joint eigenvector $f$ if and only if

$$
A_{\psi}^{(i) *} f_{\psi+\varepsilon_{i}}=\lambda_{i} f_{\psi} \quad \text { for } \psi \in G_{0}, i=1,2 .
$$

An immediate consequence is

LEMma 4.3. Let $T_{1}, T_{2}$ be as in Example 3.5 and let $f=\sum_{\psi \in G_{0}} f_{\psi} e_{\psi} \in$ $\mathbf{K}_{0}$. Then $\lambda=\left(\lambda_{1}, \lambda_{2}\right)$ is a joint eigenvalue for $T_{1}^{*}, T_{2}^{*}$ with a joint eigenvector $f$ if and only if

$$
\left(S_{\psi}^{\phi-\psi}\right)^{*} f_{\phi}=\lambda^{\phi-\psi} f_{\psi} \quad \text { for } \phi \geq \psi \in G_{0} .
$$

As a consequence, for $T_{1}, T_{2}$ as in (3.1), we have

Remark 4.4. Let $\phi \in G$ and $f=\sum_{\psi \in G_{\phi}} f_{\psi}(\cdot) e_{\psi} \in L_{\phi}$. The operators $\left(\left.T_{1}\right|_{L_{\phi}}\right)^{*},\left(\left.T_{2}\right|_{L_{\phi}}\right)^{*}$ have a joint eigenvalue $\lambda$ with a joint eigenvector $f$ if and only if

$$
S_{\eta}^{\psi-\eta}(\cdot)^{*} f_{\psi}(\cdot)=\lambda^{\psi-\eta} f_{\eta}(\cdot) \quad \text { for } \psi \geq \eta, \psi, \eta \in G_{\phi} .
$$

For the proof it is enough to note that

$$
L_{\phi}=L^{2}\left(\bigoplus_{\psi \in G_{\phi}} H_{\psi},\left.\mathcal{B}\right|_{E_{\phi}},\left.\mu\right|_{E_{\phi}}\right) \cong \bigoplus_{\psi \in G_{\phi}} L^{2}\left(H_{\psi},\left.\mathcal{B}\right|_{E_{\phi}},\left.\mu\right|_{E_{\phi}}\right) .
$$

The following lemma will be of use later.

Lemma 4.5. Let $\lambda \in \sigma_{\mathrm{p}}\left(\left(\left.T_{1}\right|_{L_{\phi_{0}}}\right)^{*},\left(\left.T_{2}\right|_{L_{\phi_{0}}}\right)^{*}\right)$. If $\phi \geq \phi_{0}$ then $\lambda \in$ $\sigma_{\mathrm{p}}\left(\left(\left.T_{1}\right|_{L_{\phi}}\right)^{*},\left(\left.T_{2}\right|_{L_{\phi}}\right)^{*}\right)$.

Proof. Let $f=\sum_{\psi \in G_{\phi_{0}}} f_{\psi}(\cdot) e_{\psi}$ be a joint eigenvector for the given eigenvalue. We define a vector $\bar{f}=\sum_{\psi \in G_{\phi}} \bar{f}_{\psi}(\cdot) e_{\psi} \in L_{\phi}$ as follows: if $\psi \in G_{\phi}$ then

$$
\bar{f}_{\psi}(X)= \begin{cases}f_{\psi}(X) & \text { if } X \in E_{\phi_{0}}, \\ 0 & \text { otherwise. }\end{cases}
$$

It is easy to see that $\bar{f} \in L_{\phi}$. Remark 4.4 shows that if $f$ is a joint eigenvector for the joint eigenvalue $\lambda$ in the space $L_{\phi_{0}}$ then so is $\bar{f}$ in the space $L_{\phi}$. 
5. Shifts defined on $\mathbf{K}_{0}$. In this section we will consider the shifts $T_{1}, T_{2} \in L\left(\mathbf{K}_{0}\right)$ defined in Example 3.5. Namely, if $f=\sum_{\phi \in G_{0}} f_{\phi} e_{\phi}$ then

$$
T_{i} f=\sum_{\phi \in G_{0}}\left(A_{\phi}^{(i)} f_{\phi}\right) e_{\phi+\varepsilon_{i}} \quad \text { for } i=1,2 .
$$

We will use the following notation: if $D \in L\left(\mathbf{K}_{0}\right)$, then there is the associated matrix $\left[D_{\alpha \beta}\right]_{\alpha, \beta \in G_{0}}$ of operators on $\mathbf{H}$ such that for $f=\sum_{\phi \in G_{0}} f_{\phi} e_{\phi}$,

$$
D f=\sum_{\alpha \in G_{0}}\left(\sum_{\beta \in G_{0}} D_{\alpha \beta} f_{\beta}\right) e_{\alpha} .
$$

As in [2], we can obtain

Lemma 5.1. Let $B=\left[B_{\alpha \beta}\right]_{\alpha, \beta \in G_{0}}$ be an operator on $\mathbf{K}_{0}$ and $\left[\gamma_{\alpha \beta}\right]_{\alpha, \beta \in G_{0}}$ be a scalar matrix such that $\left[\gamma_{\alpha \beta}\right]_{\alpha, \beta=(0,0)}^{(n, n)}$ defines a positive operator on $\mathbb{C}^{n} \times \mathbb{C}^{n}$ such that $\gamma=\sup _{\alpha \in G_{0}} \gamma_{\alpha \alpha}<\infty$. Then the matrix $\left[\gamma_{\alpha \beta} B_{\alpha \beta}\right]_{\alpha, \beta \in G_{0}}$ defines an operator $D$ on $\mathbf{K}_{0}$ satisfying $\|D\| \leq \gamma\|B\|$.

We also have the following

Lemma 5.2. The matrix $C_{n}=\left[\gamma_{\alpha \beta}\right]_{\alpha, \beta \in G_{0}}$, for $\alpha=(k, l), \beta=(i, j)$, with

$$
\gamma_{\alpha \beta}= \begin{cases}\left(1-\frac{|k-l|}{n+1}\right)\left(1-\frac{|i-j|}{n+1}\right) & \text { if }|k-l| \leq n \text { and }|i-j| \leq n, \\ 0 & \text { otherwise, }\end{cases}
$$

is positive definite.

Proof. The matrix $B_{n}=\left[b_{i j}\right], i, j \geq 0$, with

$$
b_{i j}= \begin{cases}1-\frac{|i-j|}{n+1} & \text { if }|i-j| \leq n, \\ 0 & \text { otherwise, }\end{cases}
$$

is positive definite by [5]. The matrix $C_{n}$ is the tensor product of $B_{n}$ by itself, thus it is also positive definite.

The next lemma is a consequence of Lebesgue's Dominated Convergence Theorem for a discrete measure.

Lemma 5.3. Let $\lambda_{k l} \geq 0$ and $\sum_{k, l=0}^{\infty} \lambda_{k l}<\infty$. Then

$$
\sum_{k, l=0}^{n}\left(\left(1-\frac{k}{n+1}\right)\left(1-\frac{l}{n+1}\right)-1\right) \lambda_{k l} \rightarrow 0 \quad \text { as } n \rightarrow \infty .
$$

In view of Proposition 2.1, the following theorem describes the WOTclosed algebra generated by two doubly commuting operator weighted shifts.

Theorem 5.4. An operator $D \in L\left(\mathbf{K}_{0}\right)$ belongs to $\mathcal{W}\left(T_{1}, T_{2}\right)$ if and only if $D_{\alpha \beta}=0$ for $\alpha \geq \beta$ and there is a sequence $\left\{\lambda_{\alpha}\right\}_{\alpha \in G_{0}}$ of scalars such that $D_{\alpha \beta}=\lambda_{\alpha-\beta} S_{\beta}^{\alpha-\beta}$ for $\alpha \geq \beta$. 
Proof. The statements (3.3) and (3.4) imply that $T^{s}, s \in G_{0}$, has the following matrix: $\left(T^{s}\right)_{\phi+s, \phi}=S_{\phi}^{s}$ for $\phi \in G_{0}$ and $\left(T^{s}\right)_{\phi \psi}=0$ otherwise. Hence, for each polynomial $p$ there is a sequence $\left\{\lambda_{\alpha}(p)\right\}_{\alpha \in G_{0}}$ of (finitely non-zero) scalars such that $p\left(T_{1}, T_{2}\right)_{\alpha \beta}=\lambda_{\alpha-\beta}(p) S_{\beta}^{\alpha-\beta}$ for $\alpha \geq \beta$ and $p\left(T_{1}, T_{2}\right)_{\alpha \beta}=0$ otherwise. Let $D \in \mathcal{W}\left(T_{1}, T_{2}\right)$. Then there is a net $\left\{p_{\omega}\left(T_{1}, T_{2}\right)\right\}$ of polynomials in $T_{1}, T_{2}\left(T_{1}, T_{2}\right.$ commute) converging in the Weak Operator Topology to $D$. Thus $\left(p_{\omega}\left(T_{1}, T_{2}\right)\right)_{\alpha \beta}$ converges to $D_{\alpha \beta}$. Hence $D$ has the desired matrix.

Conversely, assume that there is a sequence $\left\{\lambda_{\alpha}\right\}_{\alpha \in G_{0}}$ of scalars such that $D_{\alpha \beta}=\lambda_{\alpha-\beta} S_{\beta}^{\alpha-\beta}$ for $\alpha \geq \beta$. Consider a sequence of polynomials in $T_{1}, T_{2}$,

$$
\begin{aligned}
p_{n}\left(T_{1}, T_{2}\right) & =\sum_{k, l=0}^{n} \gamma_{k l}^{(n)} \lambda_{(k, l)} T_{1}^{k} T_{2}^{l}, \quad \text { where } \\
\gamma_{k l}^{(n)} & =\left(1-\frac{k}{n+1}\right)\left(1-\frac{l}{n+1}\right) .
\end{aligned}
$$

Lemmas 5.1 and 5.2 show that

$$
\left\|p_{n}\left(T_{1}, T_{2}\right)\right\| \leq\|D\| .
$$

Let $x \in \mathbf{H}, \zeta \in G_{0}$ and $\delta_{\zeta \beta}$ be the Kronecker $\delta$. Then

$$
\begin{aligned}
\left\|p_{n}\left(T_{1}, T_{2}\right) x e_{\zeta}-D x e_{\zeta}\right\|^{2} & \left\|\sum_{\alpha \in G_{0}}\left(\sum_{\beta \in G_{0}} \delta_{\zeta \beta}\left(p_{n}\left(T_{1}, T_{2}\right)_{\alpha \beta}\right) x\right) e_{\alpha}-\sum_{\alpha \in G_{0}}\left(\sum_{\beta \in G_{0}} \delta_{\zeta \beta} D_{\alpha \beta} x\right) e_{\alpha}\right\|^{2} \\
= & \sum_{\alpha \in G_{0}}\left\|\left(p_{n}\left(T_{1}, T_{2}\right)_{\alpha \zeta}\right) x-D_{\alpha \zeta} x\right\|^{2} \\
= & \sum_{\alpha \in G_{0}}\left\|\left(p_{n}\left(T_{1}, T_{2}\right)_{\alpha+\zeta, \zeta}\right) x-D_{\alpha+\zeta, \zeta} x\right\|^{2} \\
= & \sum_{k, l=0}^{n}\left\|\gamma_{k l}^{(n)} \lambda_{(k, l)} S_{\zeta}^{(k, l)} x-\lambda_{(k, l)} S_{\zeta}^{(k, l)} x\right\|^{2}+\sum_{\alpha \notin(n, n)}\left\|D_{\alpha+\zeta, \zeta} x\right\|^{2} \\
= & \sum_{k, l=0}^{n}\left(\gamma_{k l}^{(n)}-1\right)\left\|\lambda_{(k, l)} S_{\zeta}^{(k, l)} x\right\|^{2}+\sum_{\alpha \succeq(n, n)}\left\|D_{\alpha+\zeta, \zeta} x\right\|^{2} \\
= & \sum_{k, l=0}^{n}\left(\gamma_{k l}^{(n)}-1\right)\left\|D_{(k, l)+\zeta, \zeta} x\right\|^{2}+\sum_{\alpha \notin(n, n)}\left\|D_{\alpha+\zeta, \zeta} x\right\|^{2} .
\end{aligned}
$$


Since $\sum_{\alpha \in G_{0}}\left\|D_{\alpha+\zeta, \zeta} x\right\|^{2}=\left\|D x e_{\zeta}\right\|^{2} \leq \infty$, by Lemma 5.3 we have $\left\|p_{n}\left(T_{1}, T_{2}\right) x e_{\zeta}-D x e_{\zeta}\right\| \rightarrow 0(n \rightarrow \infty)$. Finally, $p_{n}\left(T_{1}, T_{2}\right) f \rightarrow D f$ on a dense set, and by $(5.5), p_{n}\left(T_{1}, T_{2}\right) \rightarrow D$ in the Strong Operator Topology.

THEOREM 5.5. Let $T_{1}, T_{2}$ be the operator weighted shifts (5.1) such that $\operatorname{ker} A_{\alpha}^{(i)}=\{0\}=\operatorname{ker} A_{\alpha}^{(i) *}$ for $\alpha \in G_{0}, i=1,2$. Assume also that $T_{1}^{*}, T_{2}^{*}$ have a non-zero joint eigenvalue. Then $\mathcal{W}\left(T_{1}, T_{2}\right)$ is reflexive.

P r o of. The main idea of the proof is taken from [2]. However, we present some parts of the proof because they are different. Moreover, we now have a pair of operator weighted shifts instead of a single one and we assume less about them.

Let $D \in L\left(\mathbf{K}_{0}\right)$ and $\operatorname{Lat}\left(T_{1}, T_{2}\right) \subset$ Lat $D$. The subspaces $L^{2}\left(\bigoplus_{t \in G_{\psi}} H_{t}\right)$ (for all $\psi \in G_{0}$ ) are invariant for $T_{1}, T_{2}$. Hence they are also invariant for $D$, and thus $D_{\phi \psi}=0$ if $\psi \not \leq \phi$.

Let $f \in H_{(0,0)}=\mathbf{H}$ and let $[f]$ denote the one-dimensional subspace generated by $f$. Then $\bigoplus_{\phi \in G_{0}} S_{0}^{\phi}[f]$ is invariant for $T_{1}, T_{2}$. Thus, if $\Lambda=$ $\left\{\lambda_{\phi}\right\}_{\phi \in G_{0}}$ is a sequence of scalars such that $\sum_{\phi \in G_{0}}\left|\lambda_{\phi}\right|^{2}\left\|S_{0}^{\phi} f\right\|^{2}<\infty$, then there is a sequence $\left\{\gamma_{\phi}(f)\right\}_{\phi \in G_{0}}$ depending on $\Lambda$ and $f$ such that

$$
D\left(\bigoplus_{\phi \in G_{0}} \lambda_{\phi} S_{0}^{\phi} f\right)=\bigoplus_{\phi \in G_{0}} \gamma_{\phi}(f) S_{0}^{\phi} f .
$$

Let $\phi \in G_{0}$ and let $\Lambda_{\phi}=\left\{\delta_{\phi \psi}\right\}_{\psi \in G_{0}}$, where $\delta_{\phi \psi}$ is the Kronecker $\delta$. Then there is $\Gamma_{\phi}=\left\{\gamma_{\phi \psi} f\right\}$ defined as above. As in [2], it can be shown that

$$
D_{\phi \psi} S_{0}^{\psi} f=\gamma_{\phi \psi}(f) S_{0}^{\phi} f \quad \text { for } f \in \mathbf{H} \text { and } \psi \leq \phi .
$$

Let $f, g \in \mathbf{H}$ be non-zero elements. Then using (5.7) we can prove as in [2] that

$$
\gamma_{\phi \psi}(f+g) S_{0}^{\phi}(f+g)=S_{0}^{\phi}\left(\gamma_{\phi \psi}(f) f+\gamma_{\phi \psi}(g) g\right) .
$$

Since ker $S_{0}^{\phi} \neq\{0\}$, we obtain

$$
\gamma_{\phi \psi}(f+g)(f+g)=\gamma_{\phi \psi}(f) f+\gamma_{\phi \psi}(g) g .
$$

Hence, if $f, g$ are linearly independent, then

$$
\gamma_{\phi \psi}(f)=\gamma_{\phi \psi}(g)
$$

If $f=\alpha g$, then using (5.7), we can show that

$$
\gamma_{\phi \psi}(f) S_{0}^{\phi} f=\gamma_{\phi \psi}(g) S_{0}^{\phi} f
$$

Thus, in this case we also have (5.8). Hence, the $\gamma_{\phi \psi}(f)$ do not depend on $f$, so $D_{\phi \psi} S_{0}^{\psi}=\gamma_{\phi \psi} S_{0}^{\phi}$. Now we will show that

$$
\gamma_{\phi \psi}=\gamma_{\phi-\psi, 0}
$$


We know that $\operatorname{Lat}\left(T_{1}^{*}, T_{2}^{*}\right) \subset \operatorname{Lat} D^{*}$. Let $g=\sum_{\phi \in G_{0}} g_{\phi} e_{\phi}$ be a joint eigenvector for an eigenvalue $\lambda=\left(\lambda_{1}, \lambda_{2}\right)$ for $T_{1}^{*}, T_{2}^{*}$. Then, for $\psi \in G_{0}$ and $f \in H_{(0,0)}=\mathbf{H}$, using Lemma 4.3, (3.3) and (5.2), we have

$$
\begin{aligned}
\left(D^{*} g, S_{0}^{\psi} f\right) & =\left(\sum_{\psi \leq \phi} D_{\phi \psi}^{*} g_{\phi}, S_{0}^{\psi} f\right)=\sum_{\psi \leq \phi}\left(D_{\phi \psi}^{*} g_{\phi}, S_{0}^{\psi} f\right) \\
& =\sum_{\psi \leq \phi}\left(g_{\phi}, D_{\phi \psi} S_{0}^{\psi} f\right)=\sum_{\psi \leq \phi}\left(g_{\phi}, \gamma_{\psi \phi} S_{0}^{\phi} f\right) \\
& =\sum_{\psi \leq \phi}\left(\bar{\gamma}_{\psi \phi} g_{\phi}, S_{\psi}^{\phi-\psi} S_{0}^{\psi} f\right)=\sum_{\psi \leq \phi}\left(\bar{\gamma}_{\psi \phi}\left(S_{\psi}^{\phi-\psi}\right)^{*} g_{\phi}, S_{0}^{\psi} f\right) \\
& =\sum_{\psi \leq \phi}\left(\bar{\gamma}_{\psi \phi} \lambda^{\phi-\psi} g_{\psi}, S_{0}^{\psi} f\right)=\sum_{\psi \leq \phi} \bar{\gamma}_{\psi \phi} \lambda^{\phi-\psi}\left(g_{\psi}, S_{0}^{\psi} f\right) .
\end{aligned}
$$

On the other hand, $[g]$ is invariant for $D^{*}$, and so there is $\gamma \in \mathbb{C}$ such that $D^{*} g=\gamma g$. Hence

$$
\left(D^{*} g, S_{0}^{\psi} f\right)=\gamma\left(g, S_{0}^{\psi} f\right)=\gamma\left(g_{\psi}, S_{0}^{\psi} f\right) .
$$

Since ker $S_{0}^{\psi *}=\{0\}$, we have $\overline{\mathcal{R}\left(S_{0}^{\psi}\right)}=\mathbf{H}$. Hence $\sum_{\psi \leq \phi} \bar{\gamma}_{\psi \phi} \lambda^{\phi-\psi}=\gamma$. Thus

$$
\sum_{\phi \in G_{0}} \bar{\gamma}_{\psi+\phi, \psi} \lambda^{\phi}=\sum_{\phi \in G_{0}} \bar{\gamma}_{\psi 0} \lambda^{\phi} .
$$

Let $\alpha=\left(\alpha_{1}, \alpha_{2}\right)$ be an existing non-zero eigenvalue for $T_{1}^{*}, T_{2}^{*}$. Then Lemma 4.1 shows that the above equality holds for all $\lambda=\left(\lambda_{1}, \lambda_{2}\right),\left|\lambda_{i}\right| \leq$ $\left|\alpha_{i}\right|, i=1,2$. Hence (5.9) is shown and

$$
D_{\phi \psi}=\gamma_{\phi-\psi} S_{\psi}^{\phi-\psi} \text {. }
$$

So, Theorem 5.4 implies that $D \in \mathcal{W}\left(T_{1}, T_{2}\right)$.

A consequence of the above and Proposition 2.1 is

Corollary 5.6. Let $T_{1}, T_{2}$ be operator weighted shifts whose weights and their adjonts have trivial kernels. Assume that $T_{1}, T_{2}$ doubly commute and $\sigma_{\mathrm{p}}\left(T_{1}^{*}, T_{2}^{*}\right) \neq\{0\}$. Then $\mathcal{W}\left(T_{1}, T_{2}\right)$ is reflexive.

6. The general situation. As an immediate consequence of Theorem 5.4, by (4.1) we obtain

Proposition 6.1. An operator $D \in L\left(L_{\phi}\right)\left(\mu\left(E_{\phi}\right) \neq 0\right)$ belongs to $\mathcal{W}\left(\left.T_{1}\right|_{L_{\phi}},\left.T_{2}\right|_{L_{\phi}}\right)$ if and only if there is $D(\cdot): E_{\phi} \rightarrow \bigoplus_{\psi \in G_{\phi}} H_{\psi}$ such that $D_{\alpha \beta}(\cdot)=0$ for $\alpha \geq \beta$ and $\alpha, \beta \geq \phi$ and there is a sequence $\left\{\lambda_{\alpha}\right\}_{\alpha \in G_{0}}$ of scalars such that $D_{\alpha \beta}(\cdot)=\lambda_{\alpha-\beta} S_{\beta}^{\alpha-\beta}(\cdot)$ for $\alpha \geq \beta \geq \phi$.

We are thus led to the following strengthening of Theorem 5.4: 
Theorem 6.2. An operator $D \in L(K)$ belongs to $\mathcal{W}\left(T_{1}, T_{2}\right)$ if and only if there is $D(\cdot): \mathbf{X} \rightarrow \mathcal{H}$ such that $D_{\alpha \beta}(\cdot)=0$ for $\alpha \nsupseteq \beta$ and there is a sequence $\left\{\lambda_{\alpha}\right\}_{\alpha \in G_{0}}$ of scalars such that $D_{\alpha \beta}(\cdot)=\lambda_{\alpha-\beta} S_{\beta}^{\alpha-\beta}(\cdot)$ for $\alpha \geq \beta$.

Proof. If $D \in \mathcal{W}\left(T_{1}, T_{2}\right)$ then $L_{\beta} \in$ Lat $D$. If $\alpha \geq \beta$ and $\mu\left(E_{\beta}\right) \neq$ 0 , then, by Proposition 6.1 for $\psi=\beta,\left.D\right|_{L_{\beta}}$ is represented by a function $D^{\beta}(\cdot): E_{\beta} \rightarrow \bigoplus_{t \in G_{\beta}} H_{t}$ and there is a sequence $\left\{\lambda_{\alpha}^{\beta}\right\}_{\alpha \in G_{0}}$ of scalars such that $D_{\alpha \beta}^{\beta}(\cdot)=\left.\lambda_{\alpha-\beta}^{\beta} S_{\beta}^{\alpha-\beta}(\cdot)\right|_{L_{\beta}}$. We need to show that the sequence does not depend on the $\psi$ chosen in Proposition 6.1. Let $\alpha \geq \beta \geq \psi, \mu\left(E_{\psi}\right) \neq 0$. Then $\left.D\right|_{L_{\psi}}$ is represented by a function $D^{\psi}(\cdot): E_{\psi} \rightarrow \bigoplus_{t \in G_{\psi}} H_{t}$ and there is also a sequence $\left\{\lambda_{\alpha}^{\psi}\right\}_{\alpha \in G_{0}}$ of scalars such that $D_{\alpha \beta}^{\psi}(\cdot)=\left.\lambda_{\alpha-\beta}^{\psi} S_{\beta}^{\alpha-\beta}(\cdot)\right|_{L_{\psi}}$. Let $0 \neq f(\cdot) \in \mathcal{H}_{\beta}$. Then

$$
\begin{aligned}
\lambda_{\alpha-\beta}^{\beta} S_{\beta}^{\alpha-\beta}(\cdot) f(\cdot) & =D_{\alpha \beta}^{\beta}(\cdot) f(\cdot)=\left.P_{H_{\alpha}} D\right|_{L_{\beta}} f(\cdot) e_{\beta}=P_{H_{\alpha}} D f(\cdot) e_{\beta} \\
& =\left.P_{H_{\alpha}} D\right|_{L_{\psi}} f(\cdot) e_{\beta}=D_{\alpha \beta}^{\psi}(\cdot) f(\cdot)=\lambda_{\alpha-\beta}^{\psi} S_{\beta}^{\alpha-\beta}(\cdot) f(\cdot) .
\end{aligned}
$$

Thus $\lambda_{\alpha}^{\beta}=\lambda_{\alpha}^{\psi}$ and the function $D_{\alpha \beta}^{\psi}$ does not depend on $\psi$ in fact. On the other hand, if $\alpha \nsupseteq \beta$, then $D_{\alpha \beta}=0$. In that case $D_{\alpha \beta}$ is decomposable and we can define $D_{\alpha \beta}(\cdot)=0$. Thus, it is easy to see that we can construct a function $D(\cdot): \mathbf{X} \rightarrow \mathcal{H}$ with the desired properties.

For the proof of the inverse implication we can construct a uniformly bounded sequence of polynomials $p\left(T_{1}, T_{2}\right)$ as in (5.4), (5.5). It converges on each $L_{\phi}, \phi \in G$. As in the proof of Lemma 5.4 and by the uniform boundedness (5.5) and Remark 3.2 it converges on the whole $\mathbf{K}$.

Now we will present the reflexivity results. We will consider the conditions:

(*) there is $\phi$ such that $\sigma_{\mathrm{p}}\left(\left(\left.T_{1}\right|_{L_{\phi}}\right)^{*},\left(\left.T_{2}\right|_{L_{\phi}}\right)^{*}\right) \neq\{0\}$

(**) for each $\phi \in G$ such that $\mu\left(E_{\phi}\right) \neq 0$, there is $\phi_{0} \leq \phi$ such that $\mu\left(E_{\phi_{0}}\right) \neq 0$ and $\sigma_{\mathrm{p}}\left(\left(\left.T_{1}\right|_{L_{\phi_{0}}}\right)^{*},\left(\left.T_{2}\right|_{L_{\phi_{0}}}\right)^{*}\right) \neq\{0\}$.

By Lemma $4.5,(* *)$ is equivalent to the condition

(***) for each $\phi \in G$ such that $\mu\left(E_{\phi}\right) \neq 0, \sigma_{\mathrm{p}}\left(\left(\left.T_{1}\right|_{L_{\phi}}\right)^{*},\left(\left.T_{2}\right|_{L_{\phi}}\right)^{*}\right) \neq\{0\}$.

Firstly, we strengthen $(*)$ to imply $(* *)$ :

(****) there is $\phi_{0} \in G$ and a non-zero joint eigenvalue $\lambda$ for $\left(\left.T_{1}\right|_{L_{\phi_{0}}}\right)^{*}$, $\left(\left.T_{2}\right|_{L_{\phi_{0}}}\right)^{*}$ such that there is an eigenvector $f \in L_{\phi_{0}}$ for the eigenvalue $\lambda$ satisfying

$$
\forall \phi \in G \quad \mu\left(E_{\phi}\right) \neq 0 \Rightarrow\|f\|_{L^{2}\left(E_{\phi},\left.\mu\right|_{E_{\phi}}\right)} \neq 0 .
$$

The following lemma, together with Lemma 4.5 , shows that $(* * * *)$ implies $(* *)$. 
Lemma 6.3. Let condition (****) be satisfied. If $\phi \leq \phi_{0}$ and $\mu\left(E_{\phi}\right) \neq 0$, then $\sigma_{\mathrm{p}}\left(\left.T_{1}\right|_{L_{\phi}}\right)^{*},\left(\left.T_{2}\right|_{L_{\phi}}\right)^{*} \neq\{0\}$.

Proof. Let $f=\sum_{\psi \in G_{\phi_{0}}} f_{\psi}(\cdot) e_{\psi}$ be an eigenvector for the eigenvalue $\lambda=\left(\lambda_{1}, \lambda_{2}\right)$ for $\left(\left.T_{1}\right|_{L_{\phi_{0}}}\right)^{*},\left(\left.T_{2}\right|_{L_{\phi_{0}}}\right)^{*}$, existing by $(* * * *)$. We define the vector $\bar{f}=\sum_{\psi \in G_{\phi}} \bar{f}_{\psi}(\cdot) e_{\psi}$ as follows:

(6.2) $\bar{f}_{\psi}(X)= \begin{cases}f_{\psi}(X) & \text { if } X \in E_{\phi} \text { and } \psi \in G_{\phi_{0}}, \\ \left(\lambda^{\phi_{0}-\psi}\right)^{-1} S_{\psi}^{\phi_{0}-\psi}(X)^{*} f_{\phi_{0}}(X) & \quad \text { if } X \in E_{\phi} \text { and } \psi \leq \phi_{0}, \\ \left(\lambda_{2}^{\phi_{0}^{(2)}-\psi^{(2)}}\right)^{-1} S_{\psi}^{\left(0, \phi_{0}^{(2)}-\psi^{(2)}\right)}(X)^{*} f_{\left(\psi(1), \phi_{0}^{(2)}\right)}(X) & \text { if } X \in E_{\phi} \text { and } \psi \not \leq \phi_{0}, \psi^{(2)}<\phi_{0}^{(2)}, \\ \left(\lambda_{1}^{\phi_{0}^{(1)}-\psi^{(1)}}\right)^{-1} S_{\psi}^{\left(\phi_{0}^{(1)}-\psi^{(1)}, 0\right)}(X)^{*} f_{\left(\phi_{0}^{(1)}, \psi^{(2)}\right)}(X) \\ 0 \\ \text { if } X \in E_{\phi} \text { and } \psi \not \leq \phi_{0}, \psi^{(1)}<\phi_{0}^{(1)}, \\ \text { if } X \notin E_{\phi} .\end{cases}$

First, we show that

$$
\sum_{\psi \in G_{\phi}}\left\|\bar{f}_{\psi}(\cdot)\right\|^{2}<\infty
$$

Since $f \in \mathbf{K}$, for given $\varepsilon>0$ there is $m$ such that

$$
\sum_{\substack{\psi \in G_{\phi_{0}} \\ \psi \unrhd \mathbf{m}}}\left\|\bar{f}_{\psi}(\cdot)\right\|^{2}<\varepsilon \quad \text { where } \mathbf{m}=(m, m) .
$$

Let $G^{i}=\left\{\psi \in G_{\phi}: \psi \notin G_{\phi_{0}}, \psi \not \mathbf{m}, \psi^{(i)} \leq m\right\}, i=1,2$. We have

$$
\sum_{\substack{\psi \in G_{\phi} \\ \psi \geq \mathbf{m}}}\left\|\bar{f}_{\psi}(\cdot)\right\|^{2} \sum_{\substack{\psi \in G_{\phi_{0}} \\ \psi \geq \mathbf{m}}}\left\|\bar{f}_{\psi}(\cdot)\right\|^{2}+\sum_{\psi \in G^{1}}\left\|\bar{f}_{\psi}(\cdot)\right\|^{2}+\sum_{\psi \in G^{2}}\left\|\bar{f}_{\psi}(\cdot)\right\|^{2} .
$$

Let us estimate the last sum:

$$
\begin{aligned}
& \sum_{\psi \in G^{2}}\left\|\bar{f}_{\psi}(\cdot)\right\|^{2}=\sum_{k=m}^{\infty} \sum_{l=\phi^{(2)}}^{\phi_{0}^{(2)}-1}\left\|\bar{f}_{(k, l)}(\cdot)\right\|^{2} \\
& \quad \leq \sum_{k=m}^{\infty} \sum_{l=\phi^{(2)}}^{\phi_{0}^{(2)}-1}\left\|\left(\lambda_{2}^{\phi_{0}^{(2)}-l}\right)^{-1} A_{(k, l)}^{(2)}(\cdot)^{*} \ldots A_{\left(k, \phi_{0}^{(2)}-1\right)}^{(2)}(\cdot)^{*} f_{\left(k, \phi_{0}^{(2)}\right)}\right\|^{2}
\end{aligned}
$$




$$
\begin{aligned}
& \leq \sum_{k=m}^{\infty} \sum_{l=\phi^{(2)}}^{\phi_{0}^{(2)}-1}\left|\lambda_{2}\right|^{2\left(l-\phi_{0}^{(2)}\right)}\left\|A_{(k, l)}^{(2)}\right\|^{2} \ldots\left\|A_{\left(k, \phi_{0}^{(2)}-1\right)}^{(2)}(\cdot)\right\|^{2}\left\|f_{\left(k, \phi_{0}^{(2)}\right)}(\cdot)\right\|^{2} \\
& \leq \sum_{k=m}^{\infty} \sum_{l=\phi^{(2)}}^{\phi_{0}^{(2)}-1}\left(|\lambda|^{l-\phi_{0}^{(2)}-1} C^{\phi_{0}^{(2)}-l}\right)^{2}\left\|f_{\left(k, \phi_{0}^{(2)}\right)}(\cdot)\right\|^{2}
\end{aligned}
$$

Thus (6.4) implies that

$$
\sum_{\psi \in G^{2}}\left\|\bar{f}_{\psi}(\cdot)\right\|^{2} \leq M_{1} \varepsilon,
$$

where $M_{1}$ is a suitable constant. In the same way we can estimate the last but one sum. Hence, if $M$ is a suitable constant then

$$
\begin{aligned}
\sum_{\substack{\psi \in G_{\phi} \\
\psi \not \mathbf{m}}}\left\|\bar{f}_{\psi}(\cdot)\right\|^{2} & =\sum_{\substack{\psi \in G_{\phi_{0}} \\
\psi \not \mathbf{m}}}\left\|\bar{f}_{\psi}(\cdot)\right\|^{2}+M \varepsilon \\
& =\sum_{\substack{\psi \in G_{\phi_{0}} \\
\psi \not \mathbf{m}}}\left\|f_{\psi}((\cdot))\right\|^{2}+M \varepsilon \leq(M+1) \varepsilon .
\end{aligned}
$$

Thus $\bar{f} \in \mathbf{K}$ and by (6.1), $\bar{f} \neq 0$.

Now, it is easy to see by (6.2) that $\bar{f} \in L_{\phi}$. Using Remark 4.4, one can see from (6.2) that $\lambda$ is a joint eigenvalue for $\left(\left.T_{1}\right|_{L_{\phi}}\right)^{*},\left(\left.T_{2}\right|_{L_{\phi}}\right)^{*}$.

The pair $\left\{T_{1}, T_{2}\right\}$ of operator weighted shifts is called of $I W$ type if the operators $A_{\phi}^{(i)}(\cdot), \phi \in G, i=1,2$, are invertible. We have the following

THEOREM 6.4. Let $\left\{T_{1}, T_{2}\right\}$ be a pair of operator weighted shifts of IW type. Assume that $(*)$ is satisfied. Then $\mathcal{W}\left(T_{1}, T_{2}\right)=\operatorname{Alg} \operatorname{Lat}\left(T_{1}, T_{2}\right) \cap$ $\left\{T_{1}, T_{2}\right\}^{\prime}$

Proof. Let $A \in\left\{T_{1}, T_{2}\right\} \subset L(\mathbf{K})$ and $\operatorname{Lat}\left(T_{1}, T_{2}\right) \subset \operatorname{Lat}(A)$. Let $\phi_{0} \in G$ be as in assumption $(*)$ and take $\phi \geq \phi_{0}$. Lemma 3.3 shows that $L_{\phi} \in$ $\operatorname{Lat}\left(T_{1}, T_{2}\right) \subset \operatorname{Lat}(A)$ and Theorem 5.5 and Lemma 4.5 imply that there is a sequence of polynomials $\eta_{n}^{\phi}$ of the operators $\left.T_{1}\right|_{L_{\phi}},\left.T_{2}\right|_{L_{\phi}}$ WOT-converging to $\left.A\right|_{L_{\phi}}$. Moreover, (5.5) shows that $\left\|\eta_{n}^{\phi}\right\| \leq\left\|\left.A\right|_{L_{\phi}}\right\| \leq\|A\|=C$. The following lemma is needed:

LEMMA 6.5. If $\phi_{0} \leq \phi \leq \psi$ and $n \in \mathbb{N}$, then $\left.\eta_{n}^{\psi}\right|_{L_{\phi}}=\left.\eta_{n}^{\phi}\right|_{L_{\psi}}$.

Proof. This is a consequence of the equalities (5.9) and (5.10). According to (5.4) the coefficients of the polynomials $\left.\eta_{n}^{\psi}\right|_{L_{\phi}},\left.\eta_{n}^{\phi}\right|_{L_{\psi}}$ depend directly on $\gamma_{\alpha}^{\phi}, \gamma_{\alpha}^{\psi}$ given in (5.10) ( $\phi$ in the superscript means that $\gamma_{\alpha}$ was constructed on $L_{\eta}$ ). If $\psi \leq \beta \leq \alpha$ and $f: \mathbf{X} \rightarrow H_{\beta}, f(X)=0$ for $X \notin E_{\phi}$, 
then

$$
\begin{aligned}
\gamma_{\alpha-\beta}^{\psi} S_{\beta}^{\alpha-\beta}(\cdot) f(\cdot) & =\left.P_{H_{\alpha}} A\right|_{L_{\psi}} f(\cdot) e_{\beta}=P_{H_{\alpha}} A f(\cdot) e_{\beta} \\
& =\left.P_{H_{\alpha}} A\right|_{L_{\phi}} f(\cdot) e_{\beta}=\gamma_{\alpha-\beta}^{\phi} S_{\beta}^{\alpha-\beta}(\cdot) f(\cdot) .
\end{aligned}
$$

Hence $\gamma_{\alpha}^{\phi}=\gamma_{\alpha}^{\psi}$, which finishes the proof of the lemma.

Now, as in [4], for $\phi \in G$ we consider the subspaces $M_{\phi}=\{f \in \mathbf{K}$ : $f(X)=0 \mu$-a.e. on $\left.\mathbf{X}-E_{\phi}\right\}$. We extend $\eta_{n}^{\phi}$ to the whole $M_{\phi}$ considering the spaces $M_{\phi}^{l}=\left\{f \in M_{\phi}: f(X) \in \bigoplus_{\alpha \in G_{1}} H_{\alpha} \mu\right.$-a.e. $\}$ for all non-negative integers $l$ and $\mathbf{l}=(l, l)$. We define

$$
\eta_{n}^{\phi} f=T_{1+\phi}^{-1} \eta_{n}^{\phi} T_{1+\phi} f \quad \text { for } f \in M_{\phi}^{l} .
$$

As in [4] it can be shown that the extension is well defined and we can extend $\eta_{n}^{\phi}$ to $M_{\phi}$ with $\left\|\eta_{n}^{\phi}\right\| \leq C$. The equality (6.5) and Lemma 9 of [4] show that Lemma 6.5 holds not only for $L_{\phi}$, but also for $M_{\phi}$. Hence, if $\phi \leq \psi$ and $n \in \mathbb{N}$, then $M_{\phi} \subset M_{\psi}$ and $\eta_{n}^{\phi}=\left.\eta_{n}^{\psi}\right|_{M_{\psi}}$.

Now, choose any strongly increasing sequence $\left\{\phi_{n}\right\} \subset G\left(\phi_{n}^{(i)}<\phi_{n+1}^{(i)}\right.$, $i=1,2$, and the $\phi_{k}^{(i)}$ are coordinates of $\phi_{k}$ ) with first element $\phi_{0}$. Then, by Lemma 9 of [4], $\overline{\bigcup_{n \in \mathbb{N}} M_{\phi_{n}}}=\mathbf{K}$. Hence we can define $\bigcup_{m} \eta_{m}^{\phi_{m}}$ and extend it to an operator $\eta_{m}$ on the whole $\mathbf{K}$, as in [4], with $\left\|\eta_{m}\right\| \leq C$. We have assumed that $A \in\left\{T_{1}, T_{2}\right\}^{\prime}$, hence we can prove, as in [4], that $\eta_{n}$ WOT-converges to $A$, and $A \in \mathcal{W}\left(T_{1}, T_{2}\right)$.

Next we will show the following:

Proposition 6.6. Let $T_{1}, T_{2}$ be a pair of operator weighted shifts of IW type. If $(* *)$ is satisfied, then $\operatorname{Alg} \operatorname{Lat}\left(T_{1}, T_{2}\right) \subset\left\{T_{1}, T_{2}\right\}^{\prime}$.

Proof. Let $\phi \in G$, and $\mu\left(E_{\phi}\right) \neq 0$. By Lemma 3.3, $L_{\phi} \in \operatorname{Lat}\left(T_{1}, T_{2}\right) \subset$ Lat $A$ for $\phi \in G$, and thus it is enough to show that

$$
\left.\left.A\right|_{L_{\phi}} T_{i}\right|_{L_{\phi}}=\left.\left.T_{i}\right|_{L_{\phi}} A\right|_{L_{\phi}} .
$$

We also have $\operatorname{Lat}\left(T_{1}\left|L_{\phi}, T_{2}\right|_{L_{\phi}}\right) \subset$ Lat $\left.A\right|_{L_{\phi}}$. The assumption (**) is equivalent to $(* * *)$. Hence, by Theorem 5.5, the algebra $\mathcal{W}\left(\left.T_{1}\right|_{L_{\phi}},\left.T_{2}\right|_{L_{\phi}}\right)$ is reflexive. So, (6.6) holds.

Hence we can state a consequence of Theorem 6.5 and Proposition 6.6:

THEOREM 6.7. Let $T_{1}, T_{2}$ be a pair of operator weighted shifts of IW type. If $(* *)($ or $(* * *))$ is satisfied, then $T_{1}, T_{2}$ is reflexive.

Remark 6.8. Theorem 6.7 can be proved directly from Theorem 6.2 without Theorem 6.5, which is of independent interest.

R e mark 6.9. For the sake of simplicity of notation, our main theorems are formulated for pairs. However, they can be easily generalized to $N$-tuples. 
7. Examples. We present a few examples.

ExAmple 7.1. Let $A_{\phi}^{(i)}=I, \phi \in G, i=1,2$, in (3.1). Then we obtain the unweighted shifts considered in [4]. The assumption (****) holds and the reflexivity of $\mathcal{W}\left(T_{1}, T_{2}\right)$ is shown as in [4].

ExAMPLE 7.2. Let $X_{1}, \ldots, X_{n}$ be diagrams and consider the operators $T_{1}^{(j)}, T_{2}^{(j)}$ given in Example 3.4 for $X=X_{j}$. Let $T_{i}=T_{i}^{(1)} \oplus \ldots \oplus T_{i}^{(n)}$, $i=1,2$. Assume that $T_{1}^{(i)}, T_{2}^{(i)}, i=1, \ldots, n$, are of IW type and there is $\phi_{i}$ such that $\sigma_{\mathrm{p}}\left(\left.T_{1}^{(j)}\right|_{K_{G_{\phi_{i}}}}\right)^{*},\left(\left.T_{2}^{(j)}\right|_{K_{G_{\phi_{i}}}}\right)^{*} \neq\{0\}$, where $K_{G_{\phi_{i}}}=\bigoplus_{\psi \in G_{\phi_{i}}} H_{\psi}$. Hence (**) holds for $T_{1}, T_{2}$, and thus $\mathcal{W}\left(T_{1}, T_{2}\right)$ is reflexive.

ExAmPle 7.3. Let $X$ be a bounded diagram (i.e. for each $\phi \in X$ there is $n$ such that $\left.\phi-n \varepsilon_{i} \notin X, i=1,2\right)$. Let $T_{1}, T_{2}$ be given by Example 3.4 for the diagram $X$. Assume that $T_{1}, T_{2}$ are of IW type and $\sigma_{\mathrm{p}}\left(T_{1}^{*}, T_{2}^{*}\right) \neq\{0\}$. It is easy to see that $(* *)$ holds, and so $\mathcal{W}\left(T_{1}, T_{2}\right)$ is reflexive.

ExAmple 7.4. Let $X_{0}=\{(i, j): i \geq 0$ or $j \geq 0\}$ and $\mu=\delta_{X_{0}}$. Let $A_{\phi}^{(i)}=a_{i}^{\left|\phi_{i}\right|} I, 0<a_{i}<1$ for $\phi=\left(\phi_{1}, \phi_{2}\right) \in X_{0}, i=1,2$. Then one can calculate that $\sigma_{\mathrm{p}}\left(\left.T_{1}\right|_{K_{0}}\right)^{*},\left(\left.T_{2}\right|_{K_{0}}\right)^{*} \neq\{0\}$, because the pair $\left.T_{1}\right|_{K_{0}},\left.T_{2}\right|_{K_{0}}$ is unitarily equivalent to the pair $B_{1} \otimes I, I \otimes B_{2}$, where $B_{i}, i=1,2$, are weighted shifts with $\sigma_{\mathrm{p}}\left(B_{i}^{*}\right) \neq\{0\}$. Hence Example 7.2 implies the reflexivity of $\mathcal{W}\left(T_{1}, T_{2}\right)$.

\section{References}

[1] N. P. Jawell and A. R. Lubin, Commuting weighted shifts and analytic function theory in several variables, J. Operator Theory 1 (1979), 207-223.

[2] A. Lambert, The algebra generated by an invertibly weighted shift, J. London Math. Soc. (2) 5 (1972), 741-747.

[3] - Unitary equivalence and reducibility of invertibly weighted shifts, Bull. Austral. Math. Soc. 5 (1971), 157-173.

[4] M. Ptak, Reflexivity of pairs of shifts, Proc. Amer. Math. Soc. 109 (1990), 409-415.

[5] A. L. Shields and L. J. Wallen, The commutant of certain Hilbert space operators, Indiana Univ. Math. J. 20 (1971), 777-788.

[6] M. Słociński, On the Wold-type decomposition of a pair of commuting isometries, Ann. Polon. Math. 37 (1980), 255-262.

INSTITUTE OF MATHEMATICS

UNIVERSITY OF AGRICULTURE

KRÓLEWSKA 6

30-045 KRAKÓW, POLAND 